\title{
Dementia, Homocysteine, C-reactive Protein, HDL Cholesterol, Kidney Function and Vitamin $D$ in the Oldest Old-Cluster Analysis
}

\author{
Renato Laks*, Lara Miguel Quirino Araújo, Clineu de Mello Almada Filho, Vanessa Cavalcante da Silva, Vania \\ D’Almeida, Frederico Molina Cohrs and Maysa Seabra Cendoroglo
}

Department of Geriatrics and Gerontology, Universidade Federal de São Paulo, Brazil

*Corresponding author: Renato Laks, Department of Geriatrics and Gerontology, Escola Paulista de Medicina, Universidade Federal de São PauloUNIFESP, Sao Paulo (SP), Brazil

Submission: 眥 June 01,2018; Published: 眥 September 05, 2018

\begin{abstract}
Background: Identifying the risk factors for dementia is very important in order to avoid the onset of illness. Some risk markers such as elevated homocysteine, 25(OH)D, CRP, HDL-Col. a renal function is very prevalent in the aged, potentially modifiable and can be risk factors for dementia.

Objectives: To evaluate through cluster analysis the relationship between cognitive function and the risk markers homocysteine, CRP, eGFR, 25(OH)D and HDL-Col., and to validate formed clusters relating them to the incidence of dementia, stroke and death.

Design: A longitudinal study of 156 Oldest-Old (age 80-95 years) free of cognitive impairment at baseline. Data applied to cluster analysis by two steps method. The formed clusters were compared with respect to demographic variables, neuro-cognitive assessments and serum biochemical markers obtained at baseline. To validate the cluster, we evaluated the incidence of dementia, stroke and occurrence of death in the period of 2010 to 2016.

Results: The initial cognitive tests among the three formed clusters differed only on the clock drawing test. During the six years follow-up $13.5 \%$ of all participants developed dementia, 5.1\% Stroke and 10.9\% died. The incidence of dementia, stroke and deaths in this period was significantly lower in cluster $2(\mathrm{p}=0.005)$, which had intermediate values of homocysteine and eGFR, and the highest CRP, HDL-Col. and 25(OH)D. The cluster 1 had the lowest eGFR, HDL-Col. and 25(OH)D, intermediate levels of CRP and the highest levels of homocysteine, and showed the highest incidence of dementia, stroke and death.
\end{abstract}

Conclusion: The combination of eGFR greater than $45 \mathrm{~mL} / \mathrm{min} / 1.73 \mathrm{~m} 2$, HDL-Col greater than 60mg/dL, 25(OH)D greater than 20mmol/L and CRP and homocysteine near their respective reference values defined a profile associated with a lower risk of dementia, stroke and death.

Keywords: Aged; 80 and over; Homocysteine; C-Reactive protein; Cholesterol; HDL; Vitamin D; Dementia

Abbreviations: MMSE: Mini Mental State Examination; GOLD: Global Initiative for Chronic Obstructive Lung Disease; COPD: Chronic Obstructive Pulmonary Disease

\section{Introduction}

The ageing of populations worldwide raises concerns about preserve functional status independence to the fullest extent of life, including physical and cognitive abilities. Dementia syndromes are highly prevalent in the elderly. However, current treatment, including acetylcholinesterase inhibitors and memantine, allows significant symptomatic improvement, but with limited effect to reduce disease progression [1]. Therefore, identifying risk factors can be very important to prevent the onset of the disease. Epidemiologic studies evaluating the individual markers homocysteine, c-reactive protein (CRP), renal function by the Estimated Glomerular Filtration Rate (eGFR), 25-hydroxyvitamin D3[25(OH)D], and HDL-cholesterol (HDL-Col) as risk factors for cognitive dysfunction have been inconclusive. One reason for this uncertainty probably include the heterogeneity of the population studied and the methodologies used. Cluster analysis should enable the evaluation of the most important variables to identify clusters with a higher risk of developing cognitive decline. In this study we followed a medically stable, non-demented community-dwelling individuals aged over 80 years. Our hypothesis was that the cluster analysis would identify distinct clusters among themselves, in relation to the risk markers: homocysteine, 25(OH)D, CRP, HDL$\mathrm{Col}$ and eGFR. And later validate the formed clusters relating them to the incidence of dementia, stroke and death in up to 6 years of follow-up.

\section{Subjects and Methods}

This is a longitudinal study. All participants gave written informed consent to participate and all protocols were approved 
by the Research Ethics Committee of the Universidade Federal de São Paulo (UNIFESP). Since 2010, medically stable non-demented community-dwelling individuals aged over 80 years are invited to participate in the study. After the first evaluation, all are followed in the Ambulatory called Longevos. For this study, the data from the first evaluation were considered, and the outcomes dementia, stroke and death were obtained by recording medical records and death certificates in the period from 2010 to 2016.

Interview, physical examination and biochemical analysis

Geriatricians and nutritionist interviewed all subjects. A structured interview recorded self-reported information on demographic details, education, medical history and current medications was administered, and data were confirmed by medical records. Physical examination was performed by our research physicians, who are also geriatricians, and by our research nutritionists and physiotherapists, all then gerontologists. Cognitive status was assessed in a variety of domains using standardized instruments. The Mini Mental State Examination (MMSE) was used as a general index of cognitive functioning [2] Cognitive Brief Screening Battery: We chose this cognitive battery for screening because it presents good accuracy for populations with lower high schooling. We used in this work mainly the item of the late evocation of the short cognitive battery because it presented sensitivity of $82.2 \%$ and specificity of $90.4 \%$ for mild Alzheimer's disease, considering scores lower than 6(2). Venous blood samples were drawn after an overnight fast. Determination of eGFR was performed according to Berlin Initiative Study Creatinine formula (BIS $\mathrm{Cr}$ ). We chose this formula because in previous work of the Longevism Project it was shown to be the best formula for GFR when cystatin is not available [3]. Outcomes To evaluate the outcomes, we consider the period from 2010 to 2016. The dementia outcome was determined according to the NIA-AA criteria, recommended by the Brazilian Association of Neurology for application in Brazil [4]. They consist of the presence of cognitive or behavioral symptoms that affect at least two cognitive domains, interfere with work ability and usual activities, and signify a decline in prior functionality, as evidenced by objective cognitive tests. The outcome of stroke was identified by the presence of neurological deficit associated with imaging examination for stroke during follow-up [5]. The death was confirmed by death certificate and records in medical records.

\section{Statistical analysis}

All statistical analysis was performed using SPSS 22.0. A descriptive analysis of the general data of the sample studied was performed. To test whether the distribution of the various variables was normal, the Kolmogorov-Smirnov test was used when $\mathrm{n}$ was greater than 50, or Shapiro-Wilk, when n was less than 50. MannWhitney nonparametric test for non-normal distribution, $\mathrm{t}$ test for normal distribution, or chi-square test for categorical variables were used for the analysis between the genders. The comparisons between the three clusters were performed using non-parametric Kruskal-Wallis test (non-normal distribution) and ANOVA (normal distribution).

\section{Cluster analysis}

The algorithm chosen was the Two Step data mining technique of partitioning type. The quality of clustering was evaluated by the silhouette coefficient.

\section{Results}

The main characteristics of the study population are summarized. The study population were predominantly female $(69.2 \%)$, with a mean age of 84.8 years (80 to 95 years). Most practice outdoor activities, with variable education very heterogeneous, according to the population of the city of São Paulo, with a mean of 4.6 years, but varying from 0-16 years of formal study. Comparing the variables between the sexes, men presented greater levels of homocysteine, triglycerides, HDL-Col., creatinine, hemoglobin, and higher scores on attention and MMSE calculation. The women had a greater number of medications in use, higher BB4 scores, chronic diseases, LDL cholesterol and eGFR. The clustering was considered satisfactory by the criterion of the silhouette. we can see that three distinct clusters were formed, with 41,55 and 60 elderly, respectively. The five risk markers used are presented in descending order of importance for the clustering process, eGFR, HDL-Col., Homocysteine, PCR and 25(OH)D. Comparing the three clusters formed, we observed statistically significant differences between them in age (with cluster 3 a little younger than the others), Triglycerides (lower in cluster 2) and Folic Acid (greater in cluster 2). Cluster 1 presented the lowest values of HDL-Col, 25(OH)D and eGFR, intermediate values of CRP and the highest values of homocysteine. Cluster two showed intermediate values of homocysteine and eGFR, and the highest values of CRP, HDL-Col. and 25(OH)D. Cluster 3 presented the lowest values of CRP and homocysteine, intermediate values of HDL-Col. and 25(OH)D, and the highest values of eGFR. Regarding performance in cognitive tests, there was a statistically significant difference only for the clock test, in which cluster 3 achieved the highest score, followed by the cluster 2 . Comparing the sexes in the follow-up, men presented a higher incidence of stroke than women $(6.3$ and $4.6 \%$, respectively), a higher number of deaths (20.8 and 6.5\%), and a higher incidence of dementia (14.6\% and 13\%), all with statistical significance $(\mathrm{p}=0.04)$. The incidence of dementia, stroke and deaths in the period from 2010 to 2015. The incidence of the 3 outcomes was significantly lower in the elderly of cluster $2(p=0.005)$.

\section{Discussion}

In this work we were able to include an expressive number of 156 Oldest-Old, with preserved functionality and free of cognitive impairment. This studied population becomes even more significant if we consider that exist just a few Brazilian studies about the Oldest-Old, and those that exist are restricted to a small number of aged people and to very specific issues [6-8]. In relation to the cluster analysis, the silhouette coefficient was positive, proving that clustering was adequate. The eGFR was the most relevant marker for clustering, followed by HDL-Col. Homocysteine and CRP also contributed to clustering, perhaps because both are inflammatory markers. 25(OH)D was the least relevant marker for clustering, although it also influenced with a weight of 0.1 . It is important 
to note that the eGFR estimated by BIS-Cr was the marker that contributed most to the formation of clusters. Even small variations in eGFR seem to have an impact on the more favorable survival [9]. In elderly patients, the association between renal function and mortality has been well studied.

Mandelli et al. [10] In a study published in 2015, accompanied 700 elderly people aged 85 years or older from the city of Biella in Italy. They evaluated the association between mortality and 5 different formulas to eGFR with mortality. Since at that age the mortality is high, all the formulas presented good correlation with mortality after 5 years. However, for the 2-year period, the two formulas with a significant association with mortality were BISCr and Cockcroft-Gault (HR 2.88 and 3.3 respectively). We can compare our results with this Italian study. The population had the same age, creatinine averages were similar $(1.0+0.5$ in Italian work and 0.98 and +0.31 in our study) and mortality was also $11.7 \%$ considering the group with the eGFR below $60 \mathrm{~mL} / \mathrm{min} / 1.73 \mathrm{~m}^{2}$ in the Italian study and $11.7 \%$ in cluster 3 of our study, which was the cluster with a mean of BIS-Cr of $60.24 \mathrm{~mL} / \mathrm{min} / 1.73 \mathrm{~m}^{2}$. Therefore, the importance of the BIS -Cr observed in our study is similar to the results obtained in a study carried out with different population and same age group, reinforcing the validity of our results. In addition, in the study published by Lopes et al. [3] we were able to compare BIS-Cr with the gold standard Ioexol, validating this formula as the one that best estimated the glomerular filtration rate in this population.

Many studies have related HDL-Col. with longevity [11] particularly those that include centenarians [12]. It was possible to observe that in this studied population, those with the highest levels of HDL-Col. also had the lowest incidence of dementia, stroke and death. Weverling-Rijnsburger in 2003, accompanied 561 85-yearolds from the city of Leiden in the Netherlands over a 4-year period. They observed mortality of $27 \%$ and incidence of stroke of $10 \%$, higher incidences than we observed in our work of $10.9 \%$ and $5.1 \%$, respectively. The highest incidence of adverse events in the Dutch study can be explained because the only inclusion criterion was to live in the city and to be 85 years old, while we included only elderly people with preserved functionality. However, in this Dutch study the lowest HDL-Col. (lower than $40 \mathrm{mg} / \mathrm{dL}$ ) was a risk factor Homocysteine values above $15 \mu \mathrm{mol} / \mathrm{L}$ are considered high [13]. We observed that the cluster with higher values of homocysteine was the one with the highest incidence of unfavorable outcomes. However, in cluster 2, even though there were elderly individuals with homocysteine above the reference values, it seems that the more adequate eGFR, HDL-Col. and 25(OH)D were more important. In this study, we selected aged who did not present acute diseases and chronic diseases were compensated. Perhaps because of this, the mean CRP values in the 3 clusters were less than $0.5 \mathrm{mg} / \mathrm{dL}$, $(61,111)$. Even so, it is interesting to note that CRP contributed to the formation of clusters, more important than 25(OH)D, but it was also higher in group 2, which evolved with a lower incidence of unfavorable outcomes. These results may be explained by the fact that the other risk markers may have been concurrent, and homocysteine and CRP levels may not have been high enough to cause any clinical repercussion.

The relationship between inflammation and adverse outcomes in the elderly has presented controversial results in the literature, we can observe this heterogeneity in two recent studies:

A. Arai et al. [14] Investigated in a longitudinal study 536 elderly people with more than 85 years and 684 centenarians in Japan, and obtained a significant relation between inflammation, dementia and mortality.

B. Metti et al. [15] Performed a longitudinal study with a 20-year follow-up, which included 905 elderly women with an average of $(88.3+2.8$ years $)$, observing 145 cases of dementia (16\%). However, women with higher levels of IL-6 had a lower incidence of dementia (OR=0.39; CI: 0.17-0.89). 25 (OH)D was the least relevant marker for clustering, although it also influenced with a weight of 0.2 . Clusters 2 and 3 presented values considered as insufficiency (less than $30 \mathrm{ng} / \mathrm{mL}$ ), and cluster 1, deficiency (less than $20 \mathrm{ng} / \mathrm{mL}$ ). We observed that there was a significant difference between the clusters, with cluster two with the highest mean of 25(OH)D, suggesting that the more adequate the values of this hormone the greater the contribution of the latter to a better survival. This influence may be related to renal function [16], bone health [17] but also of other systems that depend on higher levels of 25(OH)D for their action [18]. When comparing performance in cognitive tests, the elderly in cluster 1 had a lower average score than the elderly in clusters 2 and 3. However, in relation to the other tests, the elderly presented similar performance. The longevity included in this study had preserved cognition in the initial evaluation but differed in relation to the risk markers, which allowed the formation of the clusters. All the elderly was followed regularly and thus it was possible to evaluate the appearance of the clinical outcomes and death. At follow-up, the incidence of stroke, death and dementia was different in the 3 clusters.

These findings validate the cluster analysis in this population, which identified: cluster 1 with higher incidence of dementia, stroke and death; Cluster 2 with lower incidence and cluster 3 with intermediate incidence in the period from 2010 to 2016. Cluster analysis consists of a method that identifies distinct phenotypes of specific abnormalities, predicting the risk of outcomes. Therefore, it is a particularly suitable technique for studying diseases and risk factors with considerable diversity. Clustering validation consists of monitoring the studied population to evaluate if the cluster analysis was able to predict the outcomes investigated. This method has been used before. Chen [19] performed a cluster analysis to investigate the relationship between different phenotypes of Chronic Obstructive Pulmonary Disease (COPD), whose Global Initiative for Chronic Obstructive Lung Disease (GOLD) classification was not able to identify, with mortality. A total of 332 men with COPD, who formed 5 clusters, were included, with cluster E presenting more symptoms, severe and frequent 
exacerbations, and significant limitation of airflow. With the followup, cluster E presented higher mortality, validating clustering and allowing a more adequate approach of this population, which was not previously identified by the GOLD criteria. However, the cluster analysis in elderly individuals to evaluate risk factors for dementia, stroke and mortality had not yet been performed, and the validation of clustering made our findings even more significant.

\section{Limitations of the study}

In this study we evaluated independent longevity which was selected for convenience. Analysis of more specific markers for inflammation, such as IL-6, could contribute to a better evaluation of inflammation, considering that CRP is less specific. Outcomes were assessed in the period from 2010 to 2016 and the follow-up ranged from 1 to 6 years.

\section{Conclusion}

In this work we performed the cluster analysis of a population of 156 elderly people with preserved functionality and identified 3 clusters. The combination of GFR greater than $45 \mathrm{~mL} / \mathrm{min} / 1.73 \mathrm{~m}^{2}$, HDL-Col greater than $60 \mathrm{mg} / \mathrm{dL}, 25(\mathrm{OH})$ Vit D greater than $20 \mathrm{ng} /$ $\mathrm{mL}$, Homocysteine and CRP near the respective reference values defined a Profile related to lower risk of dementia, stroke and death.

\section{References}

1. Anand R, Gill KD, Mahdi AA (2014) Therapeutics of Alzheimer's disease: Past, present and future. Neuropharmacology 76 Pt A: 27-50.

2. Nitrini R, Caramelli P, Bottino CM, Damasceno BP, Brucki SM, et al (2005) Diagnosis of Alzheimer's disease in Brazil: cognitive and functional evaluation: Recommendations of the Scientific Department of Cognitive Neurology and Aging of the Brazilian Academy of Neurology. Arq Neuropsiquiatr 63(3A): 720-727.

3. Lopes MB, Araujo LQ Passos MT, Nishida SK, Kirsztajn GM, et al. (2013) Estimation of glomerular filtration rate from serum creatinine and cystatin C in octogenarians and nonagenarians. BMC Nephrol 14: 265.

4. Silva ABD, Ferraz AC, Pádua ACD, Teixeira AL, Massaro AR, et al. (2011) Recomendações para o diagnóstico e tratamento da doença de Alzheimer e demência vascular. Dement Neuropsychol 5(1):1-4.

5. Gagliardi RJ (2004) A investigação na fase aguda do acidente vascular cerebral (AVC). Revista da Associação Médica Brasileira 50(2): 109-126.

6. Kumar V, Tan P, Steinbach M (2006) Cluster analysis: Basic concepts and algorithms. Introduction to Data Mining. pp. 487-586.
7. Lourenço TM, Lenardt MH, Kletemberg DF, Seima MD, Tallmann AEC, et al. (2012) Capacidade funcional no idoso longevo: uma revisão integrativa. Rev Gaúcha Enferm 33(2): 176-185.

8. Galete LB (2012) UM Método Para Agrupamento EM Fluxo DE Dados Utilizando 0 Algoritmo SOM. Curitiba-PR: Pontifícia Universidade Católica do Paraná.

9. Shastri S, Katz R, Rifkin DE, Fried LF, Odden MC, et al. (2012) Kidney function and mortality in octogenarians: Cardiovascular Health Study All Stars. J Am Geriatr Soc 60(7): 1201-1207.

10. Mandelli S, Riva E, Tettamanti M, Detoma P, Giacomin A, et al. (2015) Mortality Prediction in the Oldest Old with Five Different Equations to Estimate Glomerular Filtration Rate: The Health and Anemia Populationbased Study. PLoS One 10(8): e0136039.

11. Milman S, Atzmon G, Crandall J, Barzilai N (2014) Phenotypes and genotypes of high density lipoprotein cholesterol in exceptional longevity. Curr Vasc Pharmacol 12(5): 690-697.

12. Barzilai N, Gabriely I, Gabriely M, Iankowitz N, Sorkin JD. (2001) Offspring of centenarians have a favorable lipid profile. J Am Geriatr Soc 49(1): 76-79.

13. Wichmann MA, Cruickshanks KJ, Carlsson CM, Chappell R, Fischer ME, et al. (2014) Long-term systemic inflammation and cognitive impairment in a population-based cohort. J Am Geriatr Soc 62(9): 1683-1691.

14. Arai Y, Martin-Ruiz CM, Takayama M, Abe Y, Takebayashi T, et al. (2015) Inflammation, but not telomere length, predicts successful ageing at extreme old age: A longitudinal study of semi-supercentenarians. EBioMedicine 2(10): 1549-1558.

15. Metti AL, Yaffe K, Boudreau RM, Ganguli M, Lopez OL, et al. (2014) Change in inflammatory markers and cognitive status in the oldestold women from the Study of Osteoporotic Fractures. J Am Geriatr Soc 62(4): 662-666.

16. Zheng Z, Shi H, Jia J, Li D, Lin S (2013) Vitamin D supplementation and mortality risk in chronic kidney disease: a meta-analysis of 20 observational studies. BMC Nephrol 14: 199.

17. Wintermeyer E, Ihle C, Ehnert S, Stockle U, Ochs G, et al. (2016) Crucial role of vitamin D in the musculoskeletal system. Nutrients 8(6): E319

18. Maeda SS, Borba VZ, Camargo MB, Silva DM, Borges JL, et al. (2014) Recommendations of the Brazilian Society of Endocrinology and Metabology (SBEM) for the diagnosis and treatment of hypovitaminosis D. Arq Bras Endocrinol Metabol 58(5): 411-433.

19. Chen CZ, Wang LY, Ou CY, Lee CH, Lin CC (2014) Using cluster analysis to identify phenotypes and validation of mortality in men with COPD. Lung 192(6): 889-896.
Creative Commons Attribution 4.0

International License

For possible submissions Click Here
Submit Article

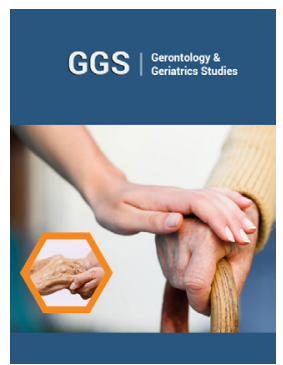

Gerontology \& Geriatrics Studies

\section{Benefits of Publishing with us}

- High-level peer review and editorial services

- Freely accessible online immediately upon publication

- Authors retain the copyright to their work

- Licensing it under a Creative Commons license

- Visibility through different online platforms 